\title{
Non-invasive Brain Stimulation for Alcohol Use Disorders: State of the Art and Future Directions
}

\author{
Noah S. Philip ${ }^{1,2}$ (1) David O. Sorensen ${ }^{1} \cdot$ Daniel M. McCalley ${ }^{3} \cdot$ Colleen A. Hanlon ${ }^{3}$ \\ Published online: 26 August 2019 \\ (C) This is a U.S. government work and its text is not subject to copyright protection in the United States; however, its text may be subject to foreign copyright \\ protection 2019
}

\begin{abstract}
Alcohol use disorders remain one of the leading causes of mortality and morbidity across the world, yet despite this impact, there are few treatment options for patients suffering from these disorders. To this end, non-invasive brain stimulation, most commonly utilizing technologies including transcranial magnetic stimulation (TMS) and transcranial direct current stimulation (tDCS), has recently emerged as promising potential treatments for alcohol use disorders. Enthusiasm for these interventions is fueled by their non-invasive nature, generally favorable safety profile, and ability to target and modulate brain regions implicated in substance use disorders. In this paper, we describe the underlying principles behind these commonly used stimulation technologies, summarize existing experiments and randomized controlled trials, and provide an integrative summary with suggestions for future areas of research. Currently available data generally supports the use of non-invasive brain stimulation as a near-term treatment for alcohol use disorder, with important caveats regarding the use of stimulation in this patient population.
\end{abstract}

Key Words Transcranial magnetic stimulation · transcranial directcurrent stimulation · non-invasive brain stimulation $\cdot$ alcohol use disorders $\cdot$ neural architecture

\section{Introduction}

Alcohol use disorders (AUD) are prevalent, devastating, and often difficult to treat. Alcohol is the third leading preventable cause of mortality and morbidity in the USA, contributing to over 80,000 deaths annually and 241 deaths every day [1]. Furthermore, the combined costs of alcohol to society were nearly 249 billion USD in 2010; to place this number in perspective, this is nearly ten times the 2016 budget of the National Institute of Health [2]. Through advances in technology, we have an unprecedented understanding of the neural circuitry involved in the alcohol use and relapse process. Yet,

Noah S. Philip

noah_philip@brown.edu

1 Center for Neurorestoration and Neurotechnology, Providence VA Medical Center, 830 Chalkstone Avenue, Providence, Rhode Island 02908, USA

2 Alpert Medical School of Brown University, Providence, Rhode Island 02903, USA

3 Department of Psychiatry, Medical University of South Carolina, Charleston, South Carolina 29412, USA despite recent advances, treatment options remain limited for AUD, and are largely constrained to pharmacological and behavioral modification paradigms. To date, there are no available treatments that directly and specifically engage the neural targets underlying AUD.

\section{Using Basic Science Knowledge to Develop an Effective, Neural Circuit-Based Treatment for AUD}

Several brain regions and circuits are involved in AUD that are potential targets for future therapeutic regimens. High relapse rates are likely due to factors that affect limbic and executive circuits in the brain, including vulnerability to salient cues and loss of cognitive control. Limbic drive and executive control are regulated by 2 cortical-subcortical neural circuits - the limbic loop that includes projections from the medial prefrontal cortex (MPFC) to the ventral striatum and the executive control loop that includes projections from the dorsolateral prefrontal cortex (DLPFC) to the dorsal striatum. Manipulation of these regions in animals has demonstrated relationships between activity in these circuits and drug-seeking behavior [3, 4]. It follows then that modulating these brain areas in patients with AUD may help decrease drinking or relapse. 
In the past, our ability to modulate neural circuits in the human brain was limited to invasive procedures such as deep brain stimulation or electroconvulsive therapy. Recently, there has been rapid expansion of non-invasive neuromodulation that can largely be grouped into 2 categories: transcranial magnetic and electrical stimulation. In this review, we will introduce each of these stimulation categories, describe their use to date in AUD with a focus on sham-controlled data, and outline directions for future growth in these areas. We then outline several key areas of innovation required to move this technology towards clinical use.

\section{Transcranial Magnetic Stimulation}

\section{Principles of TMS}

Transcranial magnetic stimulation (TMS) is a non-invasive neuromodulation technique that uses electromagnetic induction to depolarize neurons. The majority of our knowledge regarding the electrophysiological effects of TMS on the brain come from studies in the motor system. When applied over the hand knob of the primary motor cortex, a single, transient pulse of current through the TMS coil induces a reliable contraction of the contralateral hand which is proportional to the amplitude of the induced electrical field [5]. The amplitude of this motor evoked potential (MEP) in the contralateral hand can be manipulated by pharmaceutical agents that affect voltage-gated sodium channels and glutamate receptors $[6$, 7]. TMS-evoked MEPs are stable within an individual yet vary widely between individuals. Nearly $70 \%$ of the interindividual variance in motor threshold (defined as the minimum TMS intensity required to generate an MEP on $50 \%$ of the trials) is accounted for by variability in scalp-to-cortex distance, given that the TMS-evoked electrical field decays exponentially with distance. The importance of scalp-tocortex distance as a TMS dosing metric is particularly important for studies evaluating TMS as a therapeutic tool for alcohol use disorders given the known atrophy that occurs following chronic alcohol use [8].

\section{Repetitive TMS}

When single pulses of TMS are delivered in a repetitive manner, the technique is referred to as rTMS. Through rTMS, it is possible to increase or decrease cortical excitability in a transient manner (reviewed in Fitzgerald et al. and Thickbroom [9, 10]). Pascual-Leone et al. [11] were the first group to demonstrate that 20 pulses at $10-\mathrm{Hz}$ and $20-\mathrm{Hz}$ stimulation over the motor cortex increased cortical excitability, whereas Chen et al. [12] demonstrated that $15 \mathrm{~min}$ of $0.9-\mathrm{Hz}$ TMS stimulation (810 pulses) decreased cortical excitability. In addition to fixed-frequency protocols (e.g., $1 \mathrm{~Hz}, 10 \mathrm{~Hz}, 20 \mathrm{~Hz}$ ), many groups are applying burst-frequency protocols such as theta burst stimulation (TBS). Originally mirrored from preclinical studies of hippocampal plasticity, human theta burst stimulation protocols generally apply $350-\mathrm{Hz}$ pulse bursts at $5 \mathrm{~Hz}$ to the cortex. The 2 most common TBS sequences used are intermittent TBS (iTBS) and continuous TBS (cTBS). The original research in this area demonstrated that 600 pulses of iTBS can increase cortical excitability whereas 600 pulses of cTBS can reduce cortical excitability [13].

\section{TMS and its Application to Clinical Disease}

Whereas the period from 1999 to 2006 was a time of tremendous growth regarding the basic effects of various TMS protocols on motor cortex excitability, from 2006 to the present day, the momentum in rTMS research has shifted towards clinical use. In 2008, rTMS was cleared by the US FDA for the treatment of pharmacoresistant major depressive disorder. In 2018, a unique type of TMS (i.e., a coil configuration called the H-coil) was cleared for obsessive-compulsive disorder for use in combination with cue-based exposure. TMS is now used in over 650 clinics in all 50 states [14], is covered by Medicare in 48 states [15], and is being rolled out across the Veterans Affairs healthcare system. The evolving availability of clinical devices and trained staff represents a latent public health resource with incredible potential. Through this network of devices, evidence-based TMS protocols for substance dependence, including AUD, could be swiftly distributed.

\section{Literature Review-TMS for AUD}

As of June 2019, there were 12 sham-controlled studies published on the use of rTMS for alcohol addiction (Table 1). Other studies, including case reports and studies that administered rTMS in alcohol-dependent patients but did not report alcohol-related findings, have also been published but are not included in the table [35-41]. Of these studies, the majority targeted the DLPFC, and fewer the MPFC (Fig. 1). All studies stimulating the DLPFC employed high-frequency rTMS ( $>$ $5 \mathrm{~Hz}$ ) protocols and applied at least 10 sessions; 6 of them targeted the right DLPFC and 4 targeted the left DLPFC. Of studies targeting the MPFC, 1 case study utilized a lowfrequency protocol, 1 utilized a high-frequency protocol, and 2 utilized cTBS.

\section{Stimulating the DLPFC in Individuals with AUD}

The first study to apply rTMS as a therapeutic intervention for individuals with AUD was done by Mishra et al. in 2010 [20] $(n=45)$. This sham-controlled study applied $10-\mathrm{Hz}$ rTMS to the right DLPFC over 10 days, yielding a significant decrease in craving scores (measured using the ACQ-NOW) immediately after treatment. A subsequent study [28] $(n=20)$ 
Table 1 Summary of published research exploring the effects of TMS for AUD

\begin{tabular}{|c|c|c|c|c|c|c|c|c|c|c|}
\hline \multirow[t]{2}{*}{ Author (year) } & \multirow{2}{*}{$\begin{array}{l}\text { Sample } \\
\text { (real, } \\
\text { sham) }\end{array}$} & \multirow[t]{2}{*}{ Site of TMS } & \multicolumn{4}{|c|}{ Dose } & \multicolumn{2}{|c|}{ Alcohol-related effect } & \multirow[t]{2}{*}{ Blind } & \multirow{2}{*}{$\begin{array}{l}\text { Active } \\
\text { sham } \\
\text { control }\end{array}$} \\
\hline & & & $\mathrm{Hz}$ & RMT & PPS & S\# & Behavioral & Bio/brain & & \\
\hline \multicolumn{11}{|l|}{ Targeting MPFC } \\
\hline De Ridder et al. (2011) [16] & 1 & Dorsal MPFC & 1 & 50 & 600 & 1 & No, craving & Yes, EEG, BOLD & No & N/A \\
\hline Ceccanti et al. (2015) [17] & $18(9,9)$ & Dorsal MPFC & 20 & 120 & 1000 & 10 & $\begin{array}{l}\text { Yes, TLFB, VAS } \\
\text { craving }\end{array}$ & N/A & $\mathrm{P}, \mathrm{T}$ & $\mathrm{Y}$ \\
\hline Hanlon et al. (2017) [18] & $24(24,24)$ & Ventral MPFC & \multicolumn{2}{|c|}{$\mathrm{cTBS}^{\mathrm{a}}$} & 3600 & 1 & No, VAS craving & Yes, BOLD & $\mathrm{P}$ & $\mathrm{Y}$ \\
\hline $\begin{array}{l}\text { Kearney-Ramos et al. } \\
\text { (2018) [19] }\end{array}$ & $24(24,24)$ & Ventral MPFC & \multicolumn{2}{|c|}{$\begin{array}{r}\text { cTBS }^{\mathrm{a}} \\
110\end{array}$} & 3600 & 1 & No, craving & Yes, BOLD & $P$ & $\mathrm{Y}$ \\
\hline \multicolumn{11}{|l|}{ Targeting DLPFC } \\
\hline Mishra et al. (2010) [20] & $45(30,15)$ & R DLPFC & 10 & 110 & 1000 & 10 & Yes, ACQ-NOW & N/A & $\mathrm{P}$ & $\mathrm{Y}$ \\
\hline Höppner et al. (2011) [21] & $19(10,9)$ & L DLPFC & 20 & 90 & 1000 & 10 & No, OCDS & N/A & $\mathrm{P}$ & $\mathrm{N}$ \\
\hline Herremans et al. (2012) [22] & $31(15,16)$ & R DLPFC & 20 & 110 & 1560 & 1 & No, OCDS & N/A & $\mathrm{P}$ & $\mathrm{N}$ \\
\hline Herremans et al. (2013) [23] & $29(29,29)$ & R DLPFC & 20 & 110 & 1560 & 2 & No, OCDS & $\mathrm{N} / \mathrm{A}$ & $\mathrm{P}$ & $\mathrm{N}$ \\
\hline Girardi et al. (2015) [24] & $20(10)$ & L DLPFC & 20 & 120 & 2200 & 20 & Yes, OCDS & N/A & No & $\mathrm{N} / \mathrm{A}$ \\
\hline Herremans et al. (2015) [25] & 26 & R DLPFC & 20 & 110 & 1560 & 15 & No, OCDS & Yes, BOLD & $\mathrm{P}$ & $\mathrm{N}$ \\
\hline $\begin{array}{l}\text { Herremans et al. (2016) } \\
{[26]^{\mathrm{c}}}\end{array}$ & 19 & R DLPFC & 20 & 110 & 1560 & 14 & Rate dependent & Yes, BOLD & $\mathrm{P}$ & $\mathrm{N}$ \\
\hline Jansen et al. (2015) [27] & $38(20,18)$ & $\begin{array}{l}\mathrm{R} \\
\text { DLPFC }\end{array}$ & 10 & 110 & 3000 & 1 & $\mathrm{No}^{\mathrm{d}}$ & Yes, BOLD & $\mathrm{P}$ & $\mathrm{N}$ \\
\hline Mishra et al. (2015) [28] & $20(10,10)$ & $\begin{array}{l}\mathrm{L} \text { or } \mathrm{R} \\
\text { DLPFC }\end{array}$ & 10 & 110 & 1000 & 10 & Yes, ACQ-NOW & N/A & $\mathrm{P}, \mathrm{T}$ & N/A \\
\hline Rapinesi et al. (2015) [29] & 11 & L DLPFC & 18 & 120 & 1980 & 20 & Yes, OCDS & N/A & No & N/A \\
\hline Del Felice et al. (2016) [30] & $17(8,9)$ & L DLPFC & 10 & 100 & 1000 & 4 & No, VAS craving & Yes, EEG & $\mathrm{P}$ & $\mathrm{N}$ \\
\hline $\begin{array}{l}\text { Addolorato et al. } \\
\text { (2017) [31] }\end{array}$ & $11(5,6)$ & L DLPFC & 10 & 100 & 1000 & 12 & Yes, TLFB & $\begin{array}{l}\text { Yes, serum } \\
\text { cortisol }\end{array}$ & $\mathrm{P}, \mathrm{T}$ & $\mathrm{Y}$ \\
\hline
\end{tabular}

Summary of peer-reviewed research exploring the effects of TMS for individuals with AUD. Columns indicate the total number of subjects, with the number receiving real or sham stimulation, respectively, in parentheses; targeted treatment location; the dose including the stimulation frequency in Hertz (Hz), stimulation strength as a percentage of resting motor threshold (RMT), total number of pulses per stimulation session (PPS), and number of sessions (S\#); statistically significant alcohol-related effects of active stimulation over sham stimulation, divided by behavioral effects and biological/ brain-related effects; blinding $(\mathrm{P}=$ patient; $\mathrm{T}=$ treater; $\mathrm{No}=$ not blinded $)$; and whether the sham condition meets criteria to be counted as an active sham control or not $(\mathrm{Y}=$ active sham; $\mathrm{N}=$ other sham control; $\mathrm{N} / \mathrm{A}=$ not sham controlled)

${ }^{a}$ Continuous theta burst stimulation (cTBS) is not described in terms of stimulation frequency in this table

${ }^{\mathrm{b}}$ Data collected from same subjects as [18], but with separate stimulation sessions

${ }^{\mathrm{c}}$ Analysis of the subjects and data reported in [25] based on status as abstinent or relapsed at follow-up; see also [32]

${ }^{\mathrm{d}}$ Reported in [33]; see also [34]

demonstrated that 10 days of $10-\mathrm{Hz}$ rTMS stimulation to either the left or right DLPFC significantly reduced craving. Subsequent studies utilizing similar protocols $(\geq 10$ days of high-frequency rTMS) have corroborated this finding (e.g., reduced craving reported in Herremans et al. [25], reduced alcohol consumption in Addolorato et al. [31], and reduced obsessive-compulsive drinking score in Rapinesi et al. [29] and Girardi et al. [24]). Important caveats to these supporting studies, however, are that Herremans et al. [25] did not include a sham condition and Addolorato et al. [31] used an H-coil TMS design which has a broader stimulation profile than other devices.

However, several studies did not find reduction in craving and/or drinking behavior with TMS. In particular,
Höppner et al. [21] $(n=19)$ applied 10 days of $20-\mathrm{Hz}$ rTMS to the left DLPFC yet did not note any significant decrease in obsessive drinking after treatment. Furthermore, other studies that used fewer TMS sessions did not find reduced craving or use [22, 23, 30].

Several rTMS studies in AUD have incorporated neuroimaging to characterize the neurobiology of their findings. Herremans et al. [25] $(n=26)$ demonstrated that 15 sessions of $20-\mathrm{Hz}$ rTMS over 4 days did not affect cue-reactivity to alcohol as measured by fMRI (i.e., blood oxygen leveldependent [BOLD] signal). However, using a focused, region-of-interest analysis in subset $(n=19)$ of participants, higher baseline BOLD signal in the left dorsal anterior cingulate (dACC) was associated with greater alcohol abstinence 
Fig. 1 Electrical effects of transcranial magnetic stimulation to the dorsolateral prefrontal cortex and medial prefrontal cortex. The DLPFC and the MPFC have been used as TMS treatment targets in individuals with AUD. By placing a standard figure-of-8 coil over the frontal pole (EEG 10-20 system coordinates; (a), an electric field is induced in the orbitofrontal and ventral medial aspects of the prefrontal cortex. Placing a figure-of8 coil over the DLPFC (F3 coordinate) induces an electric field that extends rostrally towards the anterior PFC and caudally towards the premotor cortex (b). The scale is the induced current strength; modeling image created using SimNIBS [42]

\section{a}
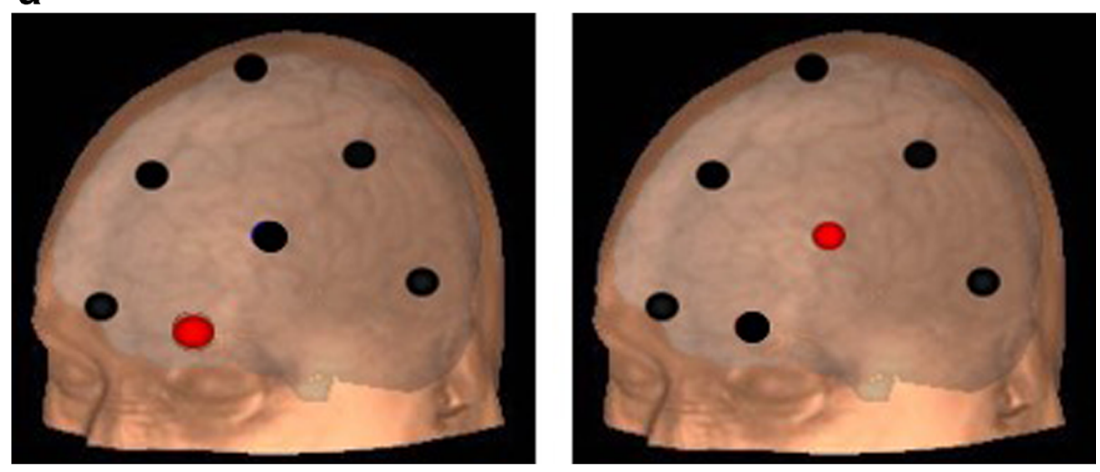

b

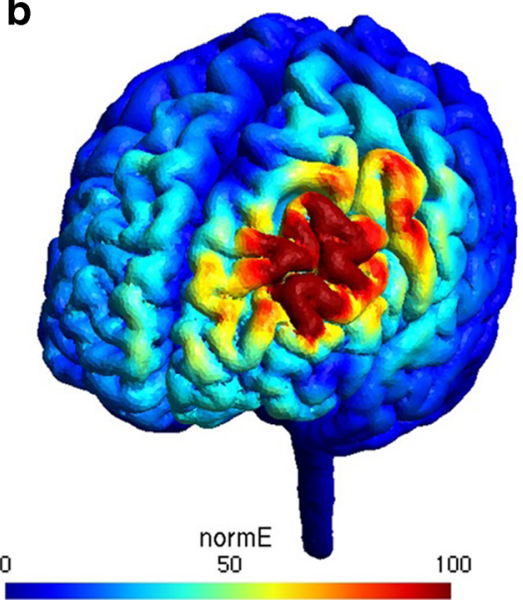

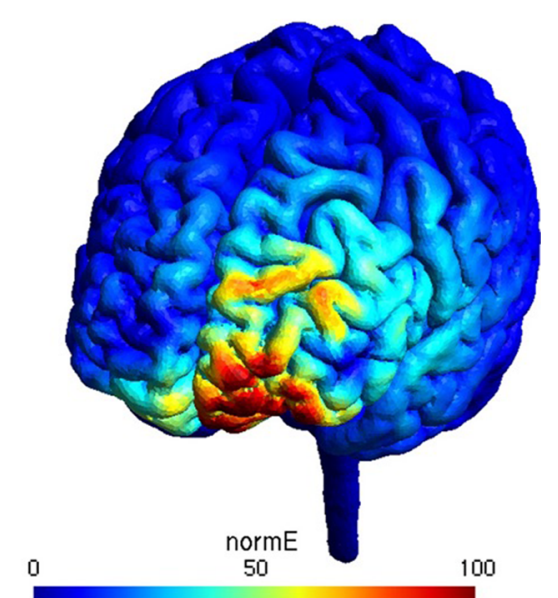

following TMS [26]. Following 10 days of $10-\mathrm{Hz}$ rTMS to the right DLPFC, Jansen et al. [27] $(n=38)$ observed increases in resting-state functional connectivity within the left frontoparietal network. Lastly, Addolorato et al. [31] $(n=11)$ revealed that individuals with AUD had higher striatal dopamine transport receptor (DAT) availability compared to healthy controls. Furthermore, 12 sessions of H-coil rTMS reduced the availability of striatal DAT.

Several studies also examined the effects of rTMS to the DLPFC on cognitive tasks related to AUD. Del Felice et al. [30] $(n=17)$ found that 4 sessions of real $10-\mathrm{Hz}$ rTMS improved performance on inhibitory control tasks (i.e., Go/No-Go, Stroop) compared to sham stimulation. Herremans et al. [23] $(n=29)$ reported a significant reduction in attentional lapses during the Go/No-Go task after real but not sham 20-Hz rTMS. Höppner et al. [21] ( $n=$ 19) examined attentional bias to alcohol cues utilizing an attentional blink paradigm after 10 daily sessions of 20Hz rTMS, finding a decrease in attentional bias to alcohol cues in individuals who received real treatment as opposed to age-matched healthy controls. Taken together, these results suggest high-frequency stimulation of the DLPFC may be a novel, efficacious strategy to enhance executive control in individuals with AUD.

\section{Stimulating the MPFC in Individuals with AUD}

An alternative strategy for improving alcohol abstinence is to decrease activity in the ventral MPFC with an attenuating form of TMS. The first study to apply rTMS to the MPFC was done by De Ridder et al. [16] $(n=1)$. In that case study, 15 days of $1-\mathrm{Hz}$ rTMS to the MPFC led to a significant reduction in craving as well as reduction in activity of the dACC and posterior cingulate cortex. Ceccanti et al. [17] $(n=18)$ applied 10 sessions of 20$\mathrm{Hz}$ rTMS to the dorsal MPFC and found significant reduction in alcohol craving associated with real stimulation that lasted for 1 month, as well as less daily alcohol consumption for 3 months. Hanlon et al. [18] $(n=25)$ demonstrated that 3600 pulses of continuous theta burst stimulation to the MPFC can reduce TMS-evoked frontalstriatal activity, and Kearney-Ramos et al. [19] demonstrated that this stimulation can decrease alcohol cuereactivity as measured by BOLD signal.

In summary, stimulation for AUD appears to be promising, but several important elements remain unclear, including optimal parameters (e.g., stimulation frequency, number of sessions and target). These are issues that are in common with other forms of stimulation for AUD. 


\section{Transcranial Direct Current Stimulation}

\section{Principles of tDCS}

Transcranial direct current stimulation is another emerging non-invasive approach to neuromodulation for AUD. In tDCS, surface electrodes, typically made up of sponges or other conductive materials, are placed on or near the head, and a small electrical current (typically $<2 \mathrm{~mA}$ ) is passed between them (Fig. 2a). This current flows from the anode (in which electricity enters the brain) to the cathode (in which electricity exits the brain). As the current travels through brain tissue, it causes small changes (generally $<1 \mathrm{mV}$ ) in neuronal membrane potentials. Scalp electrode placements are typically described using the International 10 to 20 naming convention for EEG electrodes (e.g., F3 corresponds to a location on the scalp over the left dorsolateral prefrontal cortex). Clinical applications of tDCS typically use stimulation durations in the tens of minutes repeated a number of times across days or weeks (reviewed in Philip et al. [44]).

The change in membrane potential produced by tDCS is related to the electrical field produced by a given electrode configuration, which can be modeled through a computer simulation (Fig. 2b), and the location and orientation of individual neurons in relation to the field. The orientation of a neuron within the electrical field determines whether the membrane potential change is more hyperpolarizing or depolarizing. If the polarity of the electrical field was reversed, the membrane potential change of a given neuron in the field would be inverted as well. It is thus classically described that the placement of the anode electrode over the brain target of interest is "excitatory," whereas a cathode electrode over the brain target of interest is "inhibitory." Although this model is likely incorrect (see Kronberg et al. [45]), it can be a useful heuristic to understand how configuration can impact neural activity.

Additionally, the changes in neuronal membrane potential produced by tDCS are subthreshold: they do not independently produce changes in neuronal firing. However, tDCS can modulate neural activity in the context of the underlying brain activity. The effects of tDCS are thus likely context dependent: what a person does whereas stimulation is applied changes the underlying brain state which changes the effected neural response (i.e., firing of action potentials and synaptic plasticity) to the electrical field produced by tDCS. This feature has been used in other areas of tDCS (e.g., [46]) and lays an important conceptual foundation for the use of tDCS to reduce craving for alcohol use disorder.

Importantly for clinical trials, robust sham tDCS setups are readily available. Many tDCS devices can be programmed to automatically turn off the current after an initial ramp-up period. Replicating the initial ramp-up period produces the same sensations produced by active tDCS administration and successfully blinds participants as to which treatment (active or sham) they are receiving (e.g., $[47,48])$. This process can be a

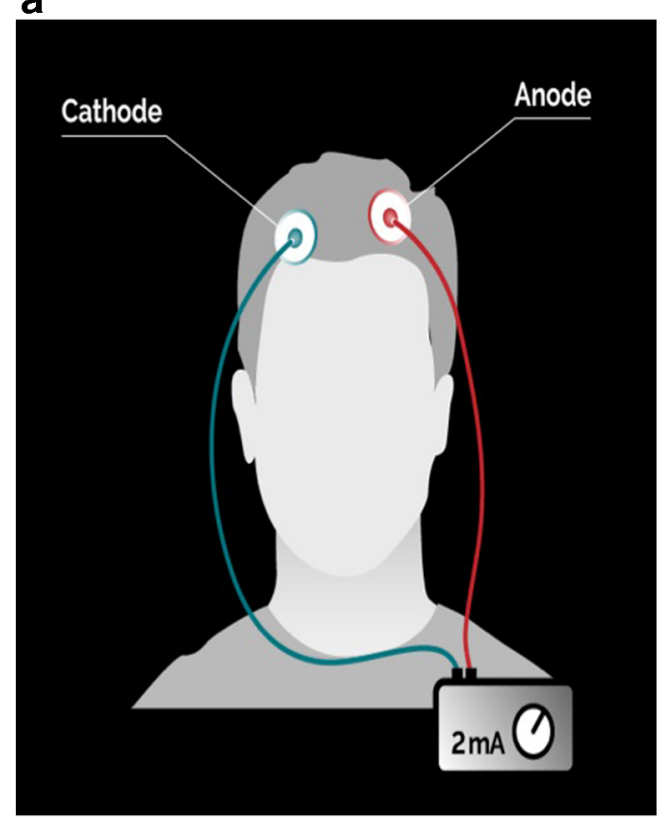

Fig. 2 a In transcranial direct current stimulation (tDCS), current $(\leq 2 \mathrm{~mA})$ is generated from a power source and enters the brain through the scalp via the anode; electrical current leaves the brain through the cathode. This results in small shifts in resting membrane potentials that can be harnessed for clinical therapeutic applications in AUD. Image

b

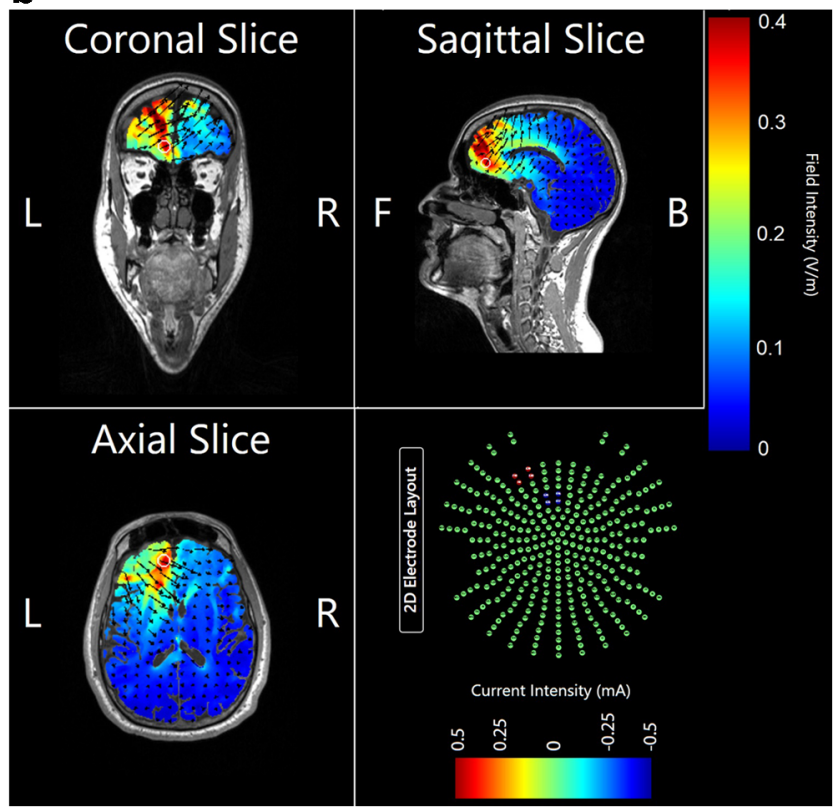

demonstrates a sample electrical field map typically used to model effects of tDCS. b In this example, $24-\mathrm{cm}$ sponges have been placed over EEG coordinates AF7 (anode) and Fz (cathode). Scale is electrical field strength in volts per meter; figure created using ROAST [43] 
double-blinded through the use of a subject-specific code that determines whether the tDCS device is in active or sham mode without alerting the administrator, allowing for robust testing of the effects of tDCS versus sham. Furthermore, there are very few inherent risks of tDCS when following safety guidelines ([49]). However, skin injuries (e.g., heating or burns) are possible due to excessive impedance, and psychiatric side effects with tDCS are also an established risk (e.g., mania; [50]).

\section{Review of tDCS for AUD}

A summary of randomized controlled trials of tDCS for the treatment of AUD can be found in Table 2. The first study investigating the efficacy of tDCS for the treatment of AUD was done by Boggio et al. $(n=13)$ [57]. Based on neuroimaging work associating DLPFC activity and alcohol craving (e.g., [63-65]), they tested tDCS in 2 configurations designed to target DLPFC (i.e., placing the anode over F3 and cathode over F4, along with the reversed placement), and found active stimulation significantly reduced craving in both configurations compared to sham. Following this initial result, others have used electrode configurations designed to target the DLPFC.

Several studies utilized the anode placed over F3. These started with Nakamura-Palacios et al. [51] $(n=49)$ which found no difference between active and sham stimulation on craving after a single tDCS session. In another study by da Silva et al. [52] $(n=13)$, active stimulation significantly decreased craving, but there was a statistical trend towards higher relapse rates during the 9 -week treatment and follow-up period in the active group. den Uyl et al. [53] $(n=41)$ tested 2 configurations: one designed to target the DLPFC and one designed to target the inferior frontal gyrus. They found significantly reduced craving scores after active tDCS using anode F3 configuration compared to sham, but stimulation targeted to the inferior frontal gyrus did not show a difference in craving scores between groups. den Uyl et al. [54] $(n=78)$ found a small reduction in cued craving following active stimulation; in another study, den Uyl et al. [55] $(n=91)$ found modest effects of active stimulation reducing 1-year relapse rates over sham stimulation, but these results were not robust to sensitivity analyses. No significant effects for craving were found. In another study, the same group [56] $(n=83)$ did not find significant effects of tDCS on craving or 1-year relapse rates.

As an alternative to placing the anode over F3, other studies placed it over F4, with cathode generally placed over F3. Klauss et al. [58] $(n=33)$ found higher abstinence rates in the 6 months following active over sham, but no effect on craving. Wietschorke et al. [59] $(n=30)$ stimulated while administering a startle paradigm to evaluate cue-reactivity to alcohol-related cues and found increased startle response following alcohol-related picture cues. Klauss et al. [60] $(n=45)$ found a significant reduction in craving scores with anodal F4 active stimulation over sham. They also found significantly lower relapse rates after 3 months in the active group compared to the sham group. Martinotti et al. [61] $(n=32)$ included AUD as a subset $(n=8)$ in a transdiagnostic study of tDCS for addictive behaviors that showed reduced craving following anodal $\mathrm{F} 4$ active stimulation over sham.

In considering context during stimulation, an interesting pattern emerges. Studies that involved the presentation of alcohol-related cues in various tasks during stimulation [51, $54-56,59]$ provide weak or no evidence of a positive effect of tDCS on alcohol-related outcomes. This is especially of interest in connection with a tDCS study done for methamphetamine use which found that tDCS reduced craving whereas stimulation was administered at rest (i.e., as monotherapy), but observed increased craving when administered during a cue-induced craving task [66]. In contrast, studies that did not present alcohol-related cues during stimulation $[52,53,57$, $58,60,61]$ all reported significant reductions in either craving, relapse rate, or both. Accordingly, at least with the existing data, the combination of alcohol-related cues with stimulation may not be ideal to improve alcohol-related outcomes. Furthermore, other stimulation parameters (current magnitude, stimulus duration, and number of sessions) vary greatly in the literature, making it difficult to draw conclusions about what parameters produce the best outcomes.

Of note, many of the studies reviewed above used modest sample sizes, and the limitations of small sample sizes on clinical trial outcomes are well known [67]. The methods for a large, multisite clinical trial protocol has been published [68]; however, data from this study is not yet publicly available. The studies reviewed here present mixed findings when considered together: about half presented absent or inconclusive evidence of a beneficial effect of tDCS. Robust testing of stimulation using parameters that have more consistently produced beneficial results (i.e., no presentation of alcoholrelated cues during stimulation, anode F4/cathode F3) should be pursued to determine whether these parameters continue to produce beneficial results, especially regarding long-term consumption and relapse outcomes. Other targets for stimulation could also be explored, as only 1 study [53] has targeted a brain region other than the DLPFC.

\section{Discussion, Considerations, and Future Directions for the Field}

Taken together, both TMS and tDCS are emerging as promising tools for the treatment of AUD, yet many important questions remain. Neither technology, with the currently available literature, appears sufficient to be recommended as a standard treatment for AUD at the current time. However, both approaches have favorable risk/benefit profiles, and have the potential to become treatments in the near future. To this 


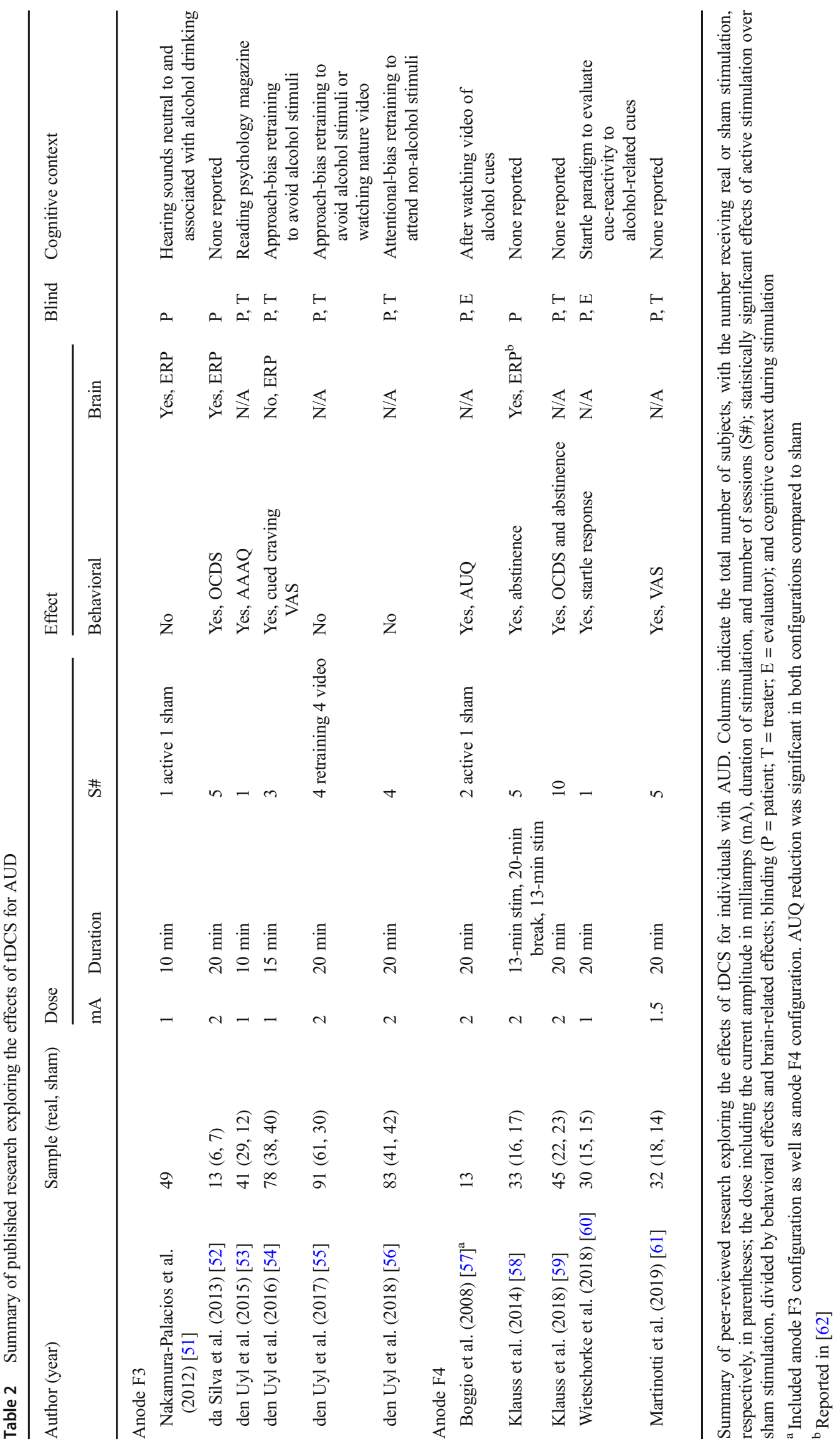


end, in this section, we review some of the near-term advances required to move this technology to clinical use.

\section{Run Studies Wherein Participants Receive 15+ Sessions of TMS or Multiple tDCS Sessions}

Most studies to date have been "Target Engagement" experiments-small clinical trials evaluating whether few stimulation sessions can induce acute changes. For TMS, none of them found an effect of stimulation on behavior, whereas the clinical trials with more sessions observed significant change in behavioral assessments. This mirrors effects observed for TMS for depression, in which a single TMS session generally does not impact mood, whereas, repeated sessions can substantively reduce clinical symptoms. Similar conclusions can be made with the tDCS literature, as the majority of studies utilize few sessions and the effects of longer term, multiple session administration remains unknown. Furthermore, important work remains to be done to evaluate longer term outcomes of stimulation for AUD.

\section{Careful Consideration of Control Conditions}

In the earlier days of TMS research, there was debate regarding the features that needed to be present in a "control" condition for TMS. Common methods used included turning the TMS coil $90^{\circ}$ (so the magnetic field was not directed towards the cortex), using an alternate brain site as a target, or no control condition at all. These strategies however generally do not take into account the somatosensory experience of TMS which can quickly unblind participants. As such, "active sham" controls need to be incorporated into future TMS studies. Furthermore, the precise role of context - and how to control for it — during tDCS remains an important and unanswered question; some studies have not controlled the context in which stimulation is applied, and in other cases the use of substance-related cues appears to have resulted in poorer outcomes.

\section{Include Standard AUD Biomarkers}

Biomarkers are not only important measurement tools to evaluate the efficacy of an intervention, but also to help classify patients into categories that are reproducible and have predictive validity. The most common clinical biomarkers used in multisite trials of alcohol use disorder are urine metabolites of alcohol, including ETG (ethyl glucuronide; a marker or recent use) and CDT (carbohydrate-deficient transferrin; a marker of cumulative use over several weeks). To date, no clinical trials of TMS or tDCS have incorporated these measures, and they will be required to compare the efficacy of non-invasive brain stimulation to other currently available AUD treatments.

\section{Consider Neural Architecture}

Understanding the potential efficacy of new treatments is predicated on the understanding of underlying mechanisms. As magnetic and electrical stimulation targets the brain, it is critical to characterize neural elements underlying any observed changes. These include consideration of the structural composition and tissue distribution in the brain, such as the distance between the TMS coil and the stimulated cortex (commonly known as "coil-to-cortex" distance) as well as gray and white matter volume, as well as proxies of neural network integrity, such as functional connectivity and white matter tract integrity. For both TMS and tDCS, electrical field modeling is also required to ensure that actual brain targets are being engaged by stimulation. These elements have largely been absent from AUD research until recently. As an example of this potential, Hanlon et al. [69] recently demonstrated that over half of the individual variance in response to active stimulation (using continuous theta burst) among individuals with AUD could be accounted for by 3 features of neural architecture - the scalp-to-cortex distance, the gray matter volume in the region stimulated, and the white matter integrity (average fractional anisotropy) along the tract from the cortical target (frontal pole) to the subcortical target (striatum). Gray matter density and white matter integrity are also important factors in TMS signal propagation among cocaine users [70]. These new insights into the role of neural architecture may be a particularly important research question for the alcohol treatment field given that heavy alcohol users have pronounced, regionally specific white matter atrophy [8].

In closing, both TMS and tDCS hold significant potential for the treatment of AUD. Other emerging forms of these technologies, such as novel patterned TMS (e.g., theta burst TMS) and transcranial alternating current or random noise stimulation, may hold additional promise for individualized treatment of AUD. If proven safe and effective, these technologies could revolutionize care for patients suffering from AUD and related substance use disorders.

Acknowledgments Effort on this manuscript was supported in part by Department of Veterans Affairs Grant I01 RX002450 and the Department of Veterans Affairs Rehabilitation Research \& Development Center for Neurorestoration and Neurotechnology (NSP, DOS), and P50 AA010761 (DMM, CAH). The authors would like to thank Logan Dowdle PhD for his contributions to the e-field model images in Fig. 1.

Required Author Forms Disclosure forms provided by the authors are available with the online version of this article.

\section{Compliance with Ethical Standards}

Conflict of Interest The authors report no biomedical conflicts of interest related to this work. Dr. Philip has received grant support from Neuronetics, Neosync and Janssen through clinical trial contracts, and has been an unpaid scientific advisory board member for Neuronetics. 
Dr. Hanlon has served as a consultant for Brainsway. Other coauthors report no conflicts of interest.

Disclaimer The funders had no role in the conduct of the study, manuscript preparation, or the decision to submit for publication. The views expressed in this article are those of the authors and do not necessarily reflect the position or policy of the NIH or Department of Veterans Affairs.

\section{References}

1. Centers for Disease Control and Prevention (US). Alcohol \& public health: alcohol-related disease impact (ARDI) [online]. Available from: https://nccd.cdc.gov/DPH_ARDI/. Accessed 5 June 2019.

2. Sacks JJ, Gonzales KR, Bouchery EE, Tomedi LE, Brewer RD. 2010 National and state costs of excessive alcohol consumption. Am J Prev Med 2015;49:e73-e9.

3. Chen BT, Yau HJ, Hatch C, Kusumoto-Yoshida I, Cho SL, Hopf FW, Bonci A. 2013 Rescuing cocaine-induced prefrontal cortex hypoactivity prevents compulsive cocaine seeking. Nature 2013; 496:359-62.

4. Stefanik MT, Moussawi K, Kupchik YM, Smith KC, Miller RL, Huff ML, Deisseroth K, Kalivas PW, LaLumiere RT. 2013 Optogenetic inhibition of cocaine seeking in rats. Addict Biol; 18: 50-3.

5. Barker AT, Freeston IL, Jabinous R, Jarratt JA. Clinical evaluation of conduction time measurements in central motor pathways using magnetic stimulation of human brain. Lancet 1986;1:1325-6.

6. Paulus W, Classen J, Cohen LG, et al. State of the art: pharmacologic effects on cortical excitability measures tested by transcranial magnetic stimulation. Brain Stimul 2008;1:151-63.

7. Ziemann U, Reis J, Schwenkreis P, et al. TMS and drugs revisited 2014. Clin Neurophysiol 2015;126:1847-68.

8. Pfefferbaum A, Sullivan EV. Disruption of brain white matter microstructure by excessive intracellular and extracellular fluid in alcoholism: evidence from diffusion tensor imaging. Neuropsychopharmacology 2005;30:423-32.

9. Fitzgerald PB, Fountain S, Daskalakis ZJ. A comprehensive review of the effects of rTMS on motor cortical excitability and inhibition. Clin Neurophysiol 2006;117:2584-96.

10. Thickbroom GW. Transcranial magnetic stimulation and synaptic plasticity: experimental framework and human models. Exp Brain Res 2007;180:583-93.

11. Pascual-Leone A, Valls-Solé J, Wassermann EM, Hallett M. Responses to rapid-rate transcranial magnetic stimulation of the human motor cortex. Brain 1994;117:847-58.

12. Chen R, Classen J, Gerloff C, et al. Depression of motor cortex excitability by low-frequency transcranial magnetic stimulation. Neurology 1997;48:1398-403.

13. Huang YZ, Edwards MJ, Rounis E, Bhatia KP, Rothwell JC. Theta burst stimulation of the human motor cortex. Neuron 2005;45:201-6.

14. Neuronetics. About NeuroStar advanced therapy [online]. Available from: https://neurostar.com/hcp/neurostar-tms-therapy/. Accessed 6 June 2019.

15. Centers for Medicare and Medicaid Services. Local coverage determinations (LCD) index by state [online]. Available from: https:// www.cms.gov/medicare-coverage-database/indexes/lcd-stateindex.aspx. Accessed 6 June 2019.

16. De Ridder D, Vanneste S, Kovacs S, Sunaert S, Dom G. Transient alcohol craving suppression by rTMS of dorsal anterior cingulate: an fMRI and LORETA EEG study. Neurosci Lett 2011;496:5-10.
17. Ceccanti M, Inghilleri M, Attilia ML, Raccah R, Fiore M, Zangen A. Deep TMS on alcoholics: effects on cortisolemia and dopamine pathway modulation. A pilot study. Can J Physiol Pharmacol 2015;93:283-90.

18. Hanlon CA, Dowdle LT, Correia B, et al. Left frontal pole theta burst stimulation decreases orbitofrontal and insula activity in cocaine users and alcohol users. Drug Alcohol Depend 2017;178: $310-7$.

19. Kearney-Ramos TE, Dowdle LT, Lench DH, et al. Transdiagnostic effects of ventromedial prefrontal cortex transcranial magnetic stimulation on cue reactivity. Biol Psychiatry Cogn Neurosci Neuroimaging 2018;3:599-609.

20. Mishra BR, Nizamie SH, Das B, Praharaj SK. Efficacy of repetitive transcranial magnetic stimulation in alcohol dependence: a shamcontrolled study. Addiction 2010;105:49-55.

21. Höppner J, Broese T, Wendler L, Berger C, Thome J. Repetitive transcranial magnetic stimulation (rTMS) for treatment of alcohol dependence. World J Biol Psychiatry 2011;12 (suppl 1):57-62.

22. Herremans SC, Baeken C, Vanderbruggen N, et al. No influence of one right-sided prefrontal HF-rTMS session on alcohol craving in recently detoxified alcohol-dependent patients: results of a naturalistic study. Drug Alcohol Depend 2012;120:209-13.

23. Herremans SC, Vanderhasselt MA, De Raedt R, Baeken C. Reduced intra-individual reaction time variability during a gonogo task in detoxified alcohol-dependent patients after one rightsided dorsolateral prefrontal HF-rTMS session. Alcohol Alcohol 2013;48:552-7.

24. Girardi P, Rapinesi C, Chiarotti F, et al. Add-on deep transcranial magnetic stimulation (dTMS) in patients with dysthymic disorder comorbid with alcohol use disorder: a comparison with standard treatment. World J Biol Psychiatry 2015;16:66-73.

25. Herremans SC, Van Schuerbeek P, De Raedt R, et al. The impact of accelerated right prefrontal high-frequency repetitive transcranial magnetic stimulation (rTMS) on cue-reactivity: an fMRI study on craving in recently detoxified alcohol-dependent patients. PLoS One 2015;10:e0136182.

26. Herremans SC, De Raedt R, Van Schuerbeek P, et al. Accelerated HF-rTMS protocol has a rate-dependent effect on dACC activation in alcohol-dependent patients: an open-label feasibility study. Alcohol Clin Exp Res 2016;40:196-205.

27. Jansen JM, van Wingen G, van den Brink W, Goudriaan AE. Resting state connectivity in alcohol dependent patients and the effect of repetitive transcranial magnetic stimulation. Eur Neuropsychopharmacol 2015;25:2230-9.

28. Mishra BR, Praharaj SK, Katshu MZ, Sarkar S, Nizamie SH. Comparison of anticraving efficacy of right and left repetitive transcranial magnetic stimulation in alcohol dependence: a randomized double-blind study. J Neuropsychiatr Clin Neurosci 2015;27:e54-9.

29. Rapinesi C, Curto M, Kotzalidis GD, et al. Antidepressant effectiveness of deep transcranial magnetic stimulation (dTMS) in patients with major depressive disorder (MDD) with or without alcohol use disorders (AUDs): a 6-month, open label, follow-up study. J Affect Disord 2015;174:57-63.

30. Del Felice A, Bellamoli E, Formaggio E, et al. Neurophysiological, psychological and behavioural correlates of rTMS treatment in alcohol dependence. Drug Alcohol Depend 2016;158:147-53.

31. Addolorato G, Antonelli M, Cocciolillo F, et al. Deep Transcranial magnetic stimulation of the dorsolateral prefrontal cortex in alcohol use disorder patients: effects on dopamine transporter availability and alcohol intake. Eur Neuropsychopharmacol 2017;27:450-61.

32. Wu G-R, Baeken C, Van Schuerbeek P, De Mey J, Bi M, Herremans SC. Accelerated repetitive transcranial magnetic stimulation does not influence grey matter volumes in regions related to alcohol relapse: an open-label exploratory study. Drug Alcohol Depend 2018;191:210-4. 
33. Jansen JM, van den Heuvel OA, van der Werf YD, et al. The effect of high-frequency repetitive transcranial magnetic stimulation on emotion processing, reappraisal, and craving in alcohol use disorder patients and healthy controls: a functional magnetic resonance imaging study. Front Psychiatry 2019;10:272.

34. Jansen JM, van den Heuvel OA, van der Werf YD, et al. Emotion processing, reappraisal, and craving in alcohol dependence: a functional magnetic resonance imaging study. Front Psychiatry 2019;10:227.

35. Rapinesi C, Kotzalidis GD, Serata D, et al. Efficacy of add-on deep transcranial magnetic stimulation in comorbid alcohol dependence and dysthymic disorder: three case reports. Prim Care Companion CNS Disord 2013;15.

36. Rapinesi C, Kotzalidis GD, Scatena P, et al. Alcohol and suicidality: could deep transcranial magnetic stimulation (dTMS) be a possible treatment? Psychiatr Danub 2014;26(3):281-4.

37. Mishra BR, Maiti R, Nizamie SH. Cerebral hemodynamics with rTMS in alcohol dependence: a randomized, sham-controlled study. J Neuropsychiatr Clin Neurosci 2016;28:319-24.

38. Qiao J, Jin G, Lei L, Wang L, Du Y, Wang X. The positive effects of high-frequency right dorsolateral prefrontal cortex repetitive transcranial magnetic stimulation on memory, correlated with increases in brain metabolites detected by proton magnetic resonance spectroscopy in recently detoxified alcohol-dependent patients. Neuropsychiatr Dis Treat 2016;12:2273-8.

39. Chung SW, Park SW, Seo YJ, Kim JH, Lee CH, Lim JY. Repetitive transcranial magnetic stimulation for Wernicke-Korsakoff Syndrome: a case report. Ann Rehabil Med 2017;41:162-6

40. Rapinesi C, Kotzalidis GD, Ferracuti S, et al. Add-on high frequency deep transcranial magnetic stimulation (dTMS) to bilateral prefrontal cortex in depressive episodes of patients with major depressive disorder, bipolar disorder I, and major depressive with alcohol use disorders. Neurosci Lett 2018;671:128-32.

41. McNeill A, Monk RL, Qureshi AW, Makris S, Heim D. Continuous theta burst transcranial magnetic stimulation of the right dorsolateral prefrontal cortex impairs inhibitory control and increases alcohol consumption. Cogn Affect Behav Neurosci 2018;18:1198-206.

42. Thielscher A, Antunes A, Saturnino GB. Field modeling for transcranial magnetic stimulation: a useful tool to understand the physiological effects of TMS? Conf Proc IEEE Eng Med Biol Soc 2015;2015:222-5.

43. Yu H, Datta A, Bikson M, Parra LC. Realistic vOlumetricApproach to Simulate Transcranial electric stimulation - ROAST - a fully automated open-source pipeline. J Neural Eng. 2019; in press.

44. Philip NS, Nelson BG, Frohlich F, Lim KO, Widge AS, Carpenter LL. Low-intensity transcranial current stimulation in psychiatry. Am J Psychiatry 2017;174:628-39.

45. Kronberg G, Bridi M, Abel T, Bikson M, Parra LC. Direct current stimulation modulates LTP and LTD: activity dependence and dendritic effects. Brain Stimul 2017;10:51-8.

46. van't Wout-Frank M, Shea MT, Larson VC, Greenberg BD, Philip NS. Combined transcranial direct current stimulation with virtual reality exposure for posttraumatic stress disorder: feasibility and pilot results. Brain Stimul. 2019;12:41-3.

47. Gandiga PC, Hummel FC, Cohen LG. Transcranial DC stimulation (tDCS): a tool for double-blind sham-controlled clinical studies in brain stimulation. Clin Neurophysiol 2006;117:845-50.

48. Ambrus GG, Al-Moyed H, Chaieb L, Sarp L, Antal A, Paulus W. The fade-in-short stimulation-fade out approach to sham tDCSreliable at $1 \mathrm{~mA}$ for naïve and experienced subjects, but not investigators. Brain Stimul 2012;5:499-504.

49. Antal A, Alekseichuk I, Bikson M, et al. Low intensity transcranial electric stimulation: safety, ethical, legal regulatory and application guidelines. Clin Neurophysiol 2017;128:1774-809.
50. Berlow YA, Zandvakili A, Carpenter LL, Philip NS. Transcranial direct current stimulation for unipolar depression and risk of treatment emergent mania: an updated meta-analysis. Brain Stimul 2019; in press.

51. Nakamura-Palacios EM, de Almeida Benevides MC, da Penha Zago-Gomes M, et al. Auditory event-related potentials (P3) and cognitive changes induced by frontal direct current stimulation in alcoholics according to Lesch alcoholism typology. Int $\mathbf{J}$ Neuropsychopharmacol 2012;15:601-16.

52. da Silva MC, Conti CL, Klauss J, et al. Behavioral effects of transcranial direct current stimulation (tDCS) induced dorsolateral prefrontal cortex plasticity in alcohol dependence. J Physiol (Paris) 2013;107:493-502.

53. den Uyl TE, Gladwin TE, Wiers RW. Transcranial direct current stimulation, implicit alcohol associations and craving. Biol Psychol 2015;105:37-42.

54. den Uyl TE, Gladwin TE, Wiers RW. Electrophysiological and behavioral effects of combined transcranial direct current stimulation and alcohol approach bias retraining in hazardous drinkers. Alcohol Clin Exp Res 2016;40:2124-33.

55. den Uyl TE, Gladwin TE, Rinck M, Lindenmeyer J, Wiers RW. A clinical trial with combined transcranial direct current stimulation and alcohol approach bias retraining. Addict Biol 2017;22:1632-40.

56. den Uyl TE, Gladwin TE, Lindenmeyer J, Wiers RW. A clinical trial with combined transcranial direct current stimulation and attentional bias modification in alcohol-dependent patients. Alcohol Clin Exp Res 2018;42:1961-9.

57. Boggio PS, Sultani N, Fecteau S, et al. Prefrontal cortex modulation using transcranial DC stimulation reduces alcohol craving: a double-blind, sham-controlled study. Drug Alcohol Depend 2008;92: 55-60.

58. Klauss J, Penido Pinheiro LC, Silva Merlo BL, et al. A randomized controlled trial of targeted prefrontal cortex modulation with tDCS in patients with alcohol dependence. Int J Neuropsychopharmacol 2014;17:1793-803.

59. Wietschorke K, Lippold J, Jacob C, Polak T, Herrmann MJ. Transcranial direct current stimulation of the prefrontal cortex reduces cue-reactivity in alcohol-dependent patients. J Neural Transm 2016;123:1173-8.

60. Klauss J, Anders QS, Felippe LV, Nitsche MA, Nakamura-Palacios EM. Multiple sessions of transcranial direct current stimulation (tDCS) reduced craving and relapses for alcohol use: a randomized placebo-controlled trial in alcohol use disorder. Front Pharmacol 2018;9:716.

61. Martinotti G, Lupi M, Montemitro C, et al. Transcranial Direct Current Stimulation Reduces Craving in Substance Use Disorders: A Double-blind, Placebo-Controlled Study. J ECT. 2019.

62. Nakamura-Palacios EM, Lopes IBC, Souza RA, et al. Ventral medial prefrontal cortex (vmPFC) as a target of the dorsolateral prefrontal modulation by transcranial direct current stimulation (tDCS) in drug addiction. J Neural Transm 2016;123:1179-94.

63. Olbrich HM, Valerius G, Paris C, Hagenbuch F, Ebert D, Juengling FD. Brain activation during craving for alcohol measured by positron emission tomography. Aust N Z J Psychiatry 2006;40:171-8.

64. Myrick H, Anton RF, Li X, et al. Differential brain activity in alcoholics and social drinkers to alcohol cues: relationship to craving. Neuropsychopharmacology 2004;29:393-402.

65. George MS, Anton RF, Bloomer C, et al. Activation of prefrontal cortex and anterior thalamus in alcoholic subjects on exposure to alcohol-specific cues. Arch Gen Psychiatry 2001;58:345-52.

66. Shahbabaie A, Golesorkhi M, Zamanian B, et al. State dependent effect of transcranial direct current stimulation (tDCS) on methamphetamine craving. Int J Neuropsychopharmacol 2014;17:1591-8. 
67. Kraemer HC, Kupfer DJ. Size of treatment effects and their importance to clinical research and practice. Biol Psychiatry 2006;59: 990-6.

68. Trojak B, Soudry-Faure A, Abello N, et al. Efficacy of transcranial direct current stimulation (tDCS) in reducing consumption in patients with alcohol use disorders: study protocol for a randomized controlled trial. Trials 2016;17:250.

69. Hanlon CA, Lench DL, Dowdle LT, Kearney-Ramos TE. Neural architecture influences rTMS-induced functional change: a DTI and FMRI study of cue-reactivity modulation in alcohol users. Clin Pharmacol Ther 2019; in press.
70. Kearney-Ramos TE, Lench DH, Hoffman M, Correia B, Dowdle LT, Hanlon CA. Gray and white matter integrity influence TMS signal propagation: a multimodal evaluation in cocaine-dependent individuals. Sci Rep 2018;8:3253.

Publisher's Note Springer Nature remains neutral with regard to jurisdictional claims in published maps and institutional affiliations. 